Supporting Information for

\title{
Uncovering Local Correlations in Polymer Electrolytes by X-ray Scattering and Molecular Dynamics Simulations
}

\author{
Whitney S. Loo ${ }^{\S, \dagger}$, Chao Fang ${ }^{\S, t, \neq}$, Nitash P. Balsara ${ }^{\dagger, \neq, \#, ~ R u i ~ W a n g}{ }^{*,+, \neq}$ \\ ${ }^{\dagger}$ Department of Chemical and Biomolecular Engineering, University of California Berkeley, Berkeley, \\ California 94720, United States \\ ${ }^{\ddagger}$ Materials Sciences Division, "Joint Center for Energy Storage Research (JCESR), Lawrence Berkeley \\ National Lab, Berkeley, California 94720, United States \\ $\S$ W.S.L. and C. F Contributed equally \\ ${ }^{*}$ Corresponding author email: ruiwang325@berkeley.edu
}




\section{I . Parameters for each simulation system}

Table S1: Simulation parameters for PEO/LiTFSI systems

\begin{tabular}{cccc}
\hline $\begin{array}{c}\boldsymbol{r} \\
\left(\left[\mathrm{Li}^{+}\right] /[\mathrm{EO}]\right)\end{array}$ & $\begin{array}{c}\boldsymbol{m} \\
(\mathbf{m o l} / \mathbf{k g})\end{array}$ & $\begin{array}{c}\text { Number of } \\
\text { LiTFSI salt } \\
\text { molecules }\end{array}$ & $\begin{array}{c}\text { Number of PEO } \\
\text { chains }\end{array}$ \\
\hline 0 & 0 & 0 & 35 \\
\hline 0.03 & 0.68 & 55 & 33 \\
\hline 0.05 & 1.13 & 88 & 32 \\
\hline 0.10 & 2.25 & 165 & 30 \\
\hline 0.15 & 3.38 & 231 & 28 \\
\hline 0.20 & 4.51 & 286 & 26 \\
\hline 0.25 & 5.63 & 330 & 24 \\
\hline 0.30 & 6.76 & 363 & 22 \\
\hline
\end{tabular}

\section{II . Equilibrium of simulation systems}

The evolution of the average radius of gyration for PEO is tracked in Figure S1 to confirm that the equilibrium state of the systems has been achieved.

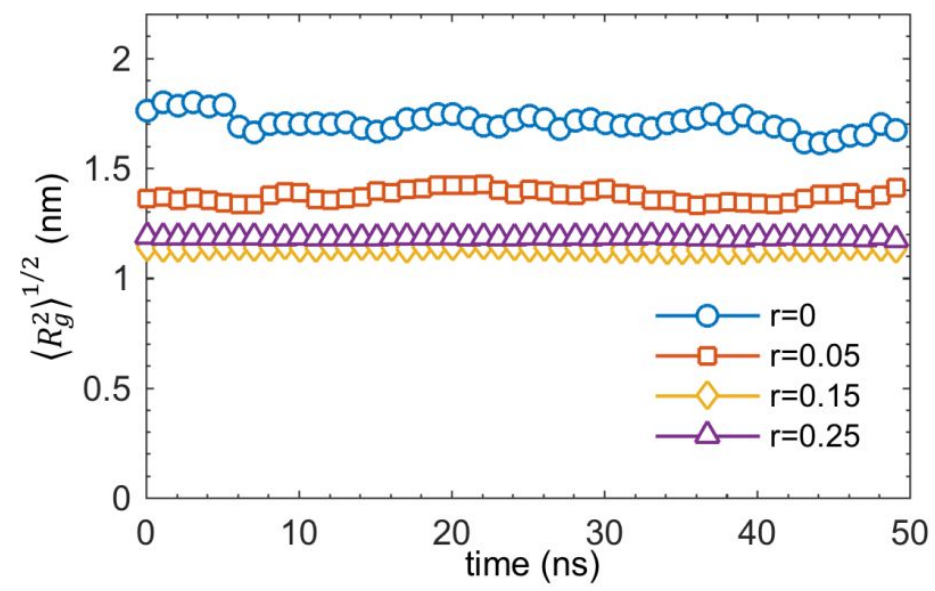

Figure S1. Evolution of the average radius of gyration of PEO chain, $\left\langle R_{g}^{2}\right\rangle^{\frac{1}{2}}$, at different salt concentrations during the last $50 \mathrm{~ns}$ after equilibrium: $r=0$ [Li+]/[EO] (after $250 \mathrm{~ns}$ ); $r=0.05$ (after $150 \mathrm{~ns}$ ); $r=0.15$ (after $150 \mathrm{~ns}$ ); $r=0.25$ (after $150 \mathrm{~ns}$ ).

\section{Total structure factors at $363.15 \mathrm{~K}$ and $368.15 \mathrm{~K}$}


The comparison of total structure factors at $363.15 \mathrm{~K}$ and $368.15 \mathrm{~K}$ shows negligible difference, e.g., the total structural factors at $r=0.1$ in Figure $\mathrm{S} 2$, suggesting that equilibrium simulations in present study at $368.15 \mathrm{~K}$ are capable of representing experimental data taken at $363.15 \mathrm{~K}$.

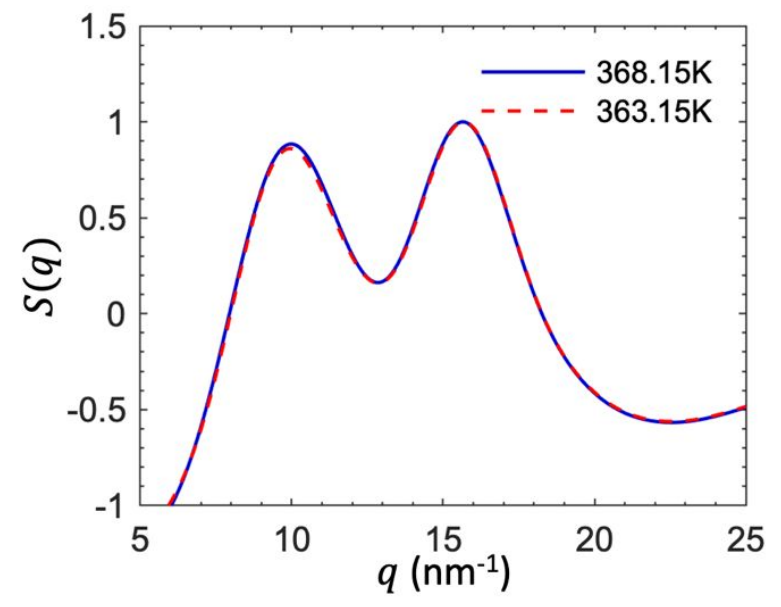

Figure S2. Total structure factors at $363.15 \mathrm{~K}$ and at $363.15 \mathrm{~K}$ at $r=0.1$.

\section{Charge-ordering from radial distributions of ions}

The charge-ordering between $\mathrm{Li}^{+}$and TFSl- at $r=0.10\left[\mathrm{Li}^{+}\right] /[\mathrm{EO}]$ is shown in Figure $\mathrm{S} 3$. There is anti-correlation between the radial distribution functions of $\mathrm{Li}^{+}-\mathrm{N}(\mathrm{TFSI})^{-}$) and $\mathrm{Li}^{+}-\mathrm{Li}^{+}$(or $\mathrm{N}\left(\mathrm{TFSI}^{-}\right)$$\mathrm{N}\left(\mathrm{TFSI}^{-}\right)$), whose periodicity align with the strong peaks and deep valley at $q=5.9 \mathrm{~nm}^{-1}$ shown in Figure $4 a$ of the main text.

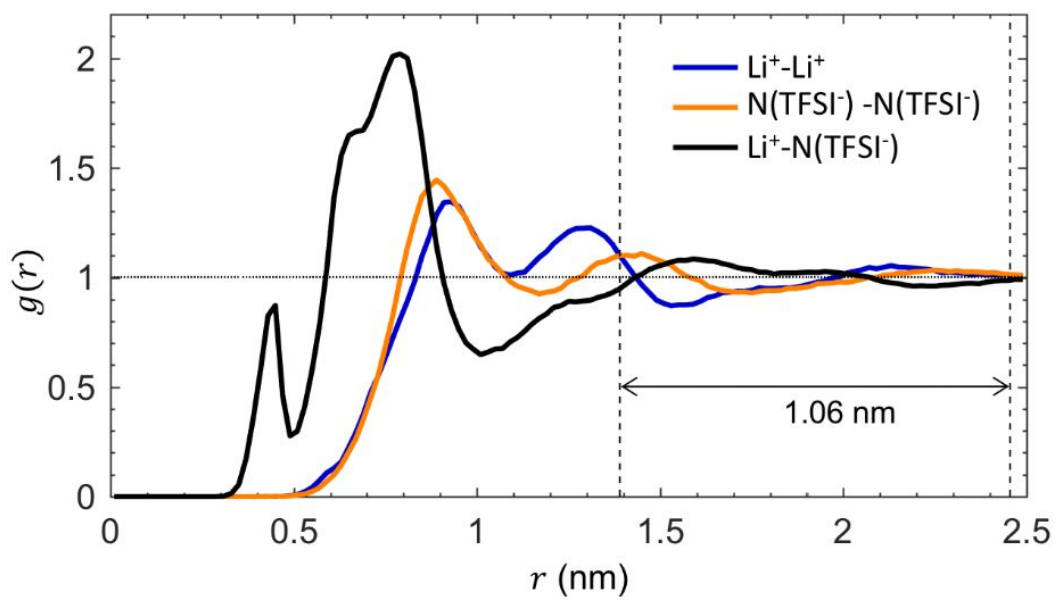

Figure S3. Radial distribution functions for $\mathrm{Li}^{+}-\mathrm{Li}^{+}, \mathrm{N}(\mathrm{TFSI}-)-\mathrm{N}(\mathrm{TFSI}-)$, and $\mathrm{Li}^{+}-\mathrm{N}(\mathrm{TFSI})$ at $r=0.1$ [ $\left.\mathrm{Li}^{+}\right] /[\mathrm{EO}]$. The two dashed lines mark the real space distance, $d=2 \pi / q^{*}=1.06 \mathrm{~nm}$, corresponding to $q^{*}=5.9 \mathrm{~nm}^{-1}$ in Figure $4 a$ of the main text. 


\section{Decomposition of total structure factor at $r=0.05$ and $r=0.20$.}

Figure S4 shows the decomposition of total structure factor for salt concentrations below and above $r=0.10\left[\mathrm{Li}^{+}\right] /[\mathrm{EO}]$. The decomposition into partial structure factors related to PEO and ions, the intermolecular and intramolecular structure factors for $\mathrm{TFSI}^{-}$, and radial distribution functions for Nitrogen atoms of $\mathrm{TFSI}^{-}$are similar to these shown in Figure 4 of the main text for $r$ $=0.10\left[\mathrm{Li}^{+}\right] /[\mathrm{EO}]$. This confirms that the same molecular interactions responsible for the two peaks hold for other salt concentrations.

(a) $r=0.05$

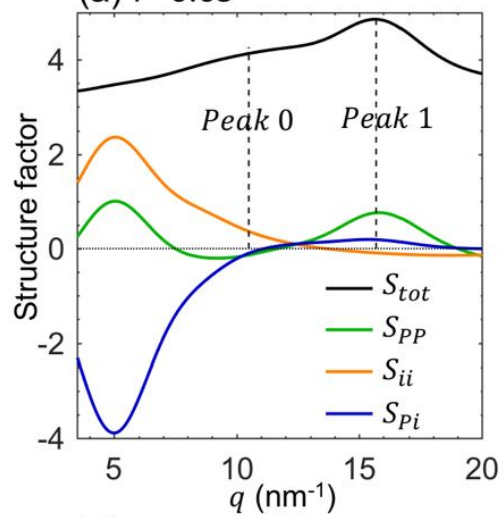

(d) $r=0.20$

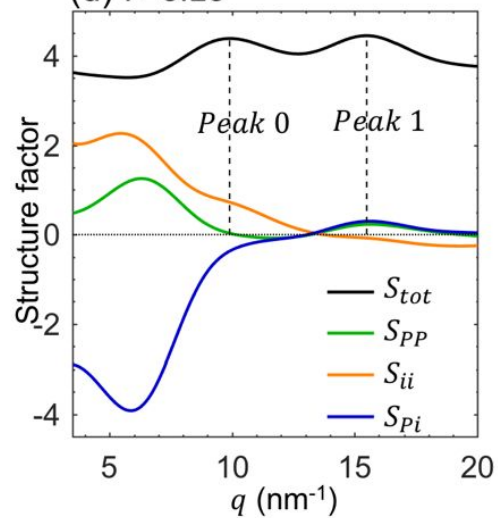

(b) $r=0.05$

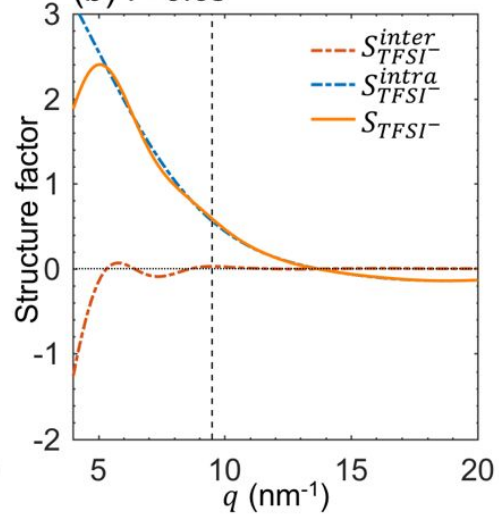

(e) $r=0.20$

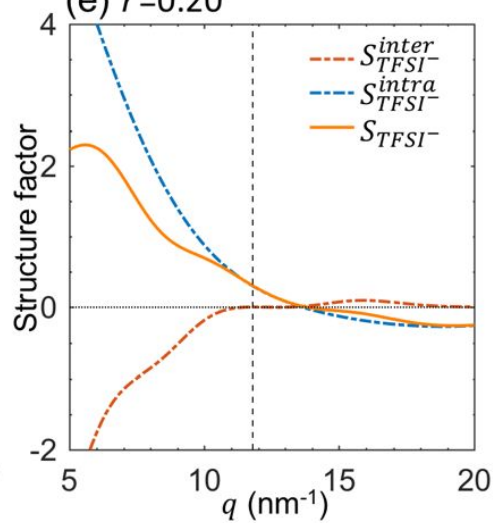

(c) $r=0.05$
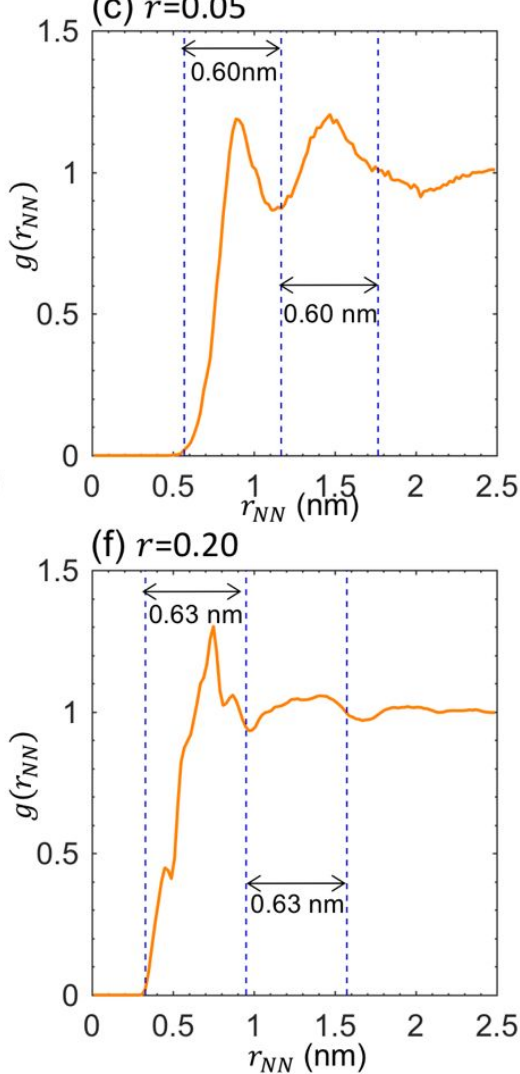

Figure S4. Structure factor of PEO/LiTFSI at $r=0.05$ (top panel) and $r=0.20$ (bottom panel). (a,d) Decomposition of total structure factor, $S_{\text {tot }}$ shown in black, from the three main contributions: PEO-PEO ( $S_{P P}$, green), ion-ion ( $S_{i i}$, orange), and PEO-ion ( $S_{P i}$, blue). The total structure factor is shifted vertically by 4 for clarity. The vertical dashed lines mark the two peaks of total structure factor (black) that are identical to the peaks in $I(q)$. (b,e) Structure factor from TFSI anions: total ( $S_{T F S I-}$, orange), the contributions from intermolecular $\left(S_{T F S I-}^{\text {inter }}\right.$, red dash-dotted line) and intramolecular interactions $\left(S_{T F S I-}^{\text {intra }}\right.$, blue dash-dotted line). The vertical dashed line marks the position of the peak from the intermolecular contributions that is responsible for Peak 0. (c,f) Radial distribution function of nitrogen atoms from TFSI', $g\left(r_{N N}\right)$, as a function of distance, $r_{N N}$, from simulations. The two groups of dashed lines mark the real space distance, $d=2 \pi / q_{0}^{*}\left(q_{0}^{*}\right.$ is the position of Peak 0$)$, starting from the onset of $g\left(r_{N N}\right)$. 


\section{Charge rescaling procedures and results}

Relative heights of scattering peaks. Figure S5 shows the fitting of reduced scattering intensities into two Gaussians at $r=0.10\left[\mathrm{Li}^{+}\right] /[\mathrm{EO}]$. The scattering intensity is first rescaled by the maximum. Then the linear base in the range between $7.5 \mathrm{~nm}^{-1}$ to the left and $4.5 \mathrm{~nm}^{-1}$ to the right of the right peak, which captures both Peak 0 and Peak 1, is cut. Thus, the reduced intensity is fit into two Gaussians with maxima $I_{l}$ and $I_{r}$ at Peak 0 and Peak 1, respectively. The relative height of Peak 0 is defined as $I_{l} /\left(I_{l}+I_{r}\right)$ and is used to quantify the deviation of the scattering profiles from simulation to WAXS experiment in partial charge rescaling.
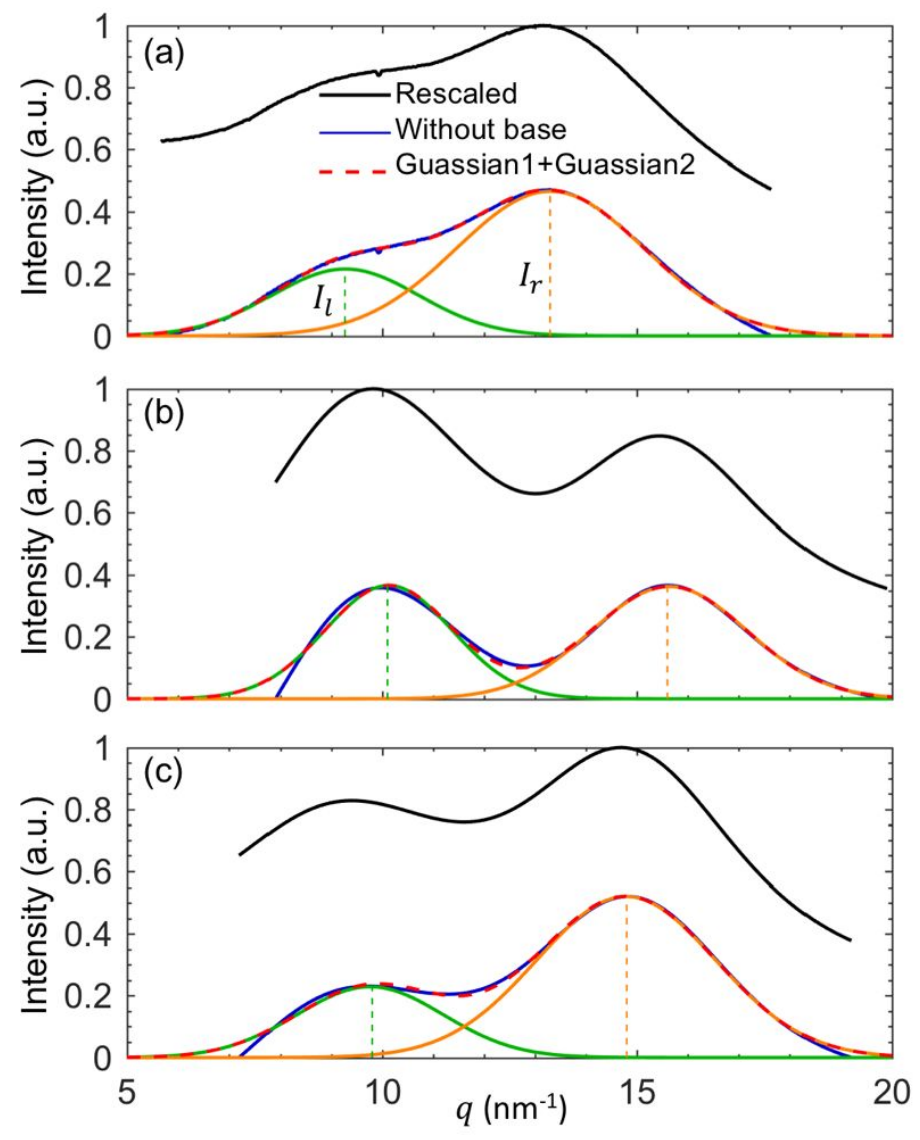

Figure S5. Relative heights of the two peaks for $r=0.10\left[\mathrm{Li}^{+}\right] /[\mathrm{EO}]$ (a) from experiment; (b) from simulation with unadjusted force field; (c) from simulation with $R_{f}=0.6$. The scattering intensities (black solid line) are rescaled based on the maximum (highest point of the black solid line). This is followed by cutting a linear base (extending from the right peak by $7.5 \mathrm{~nm}^{-1}$ to the left and by $4.5 \mathrm{~nm}^{-1}$ to the right, blue solid line). The reduced intensities are fitted into two Gaussians (green and orange solid lines), the sum of which are shown in red dashed lines. The two vertical dashed lines denote the maxima at the two peaks (left peak: $I_{l}$; right peak: $I_{r}$ ). 
Adjusting ionic partial charges only. We first applied a charge rescaling factor on LiTFSI, as is usually done in modeling concentrated polymer electrolytes. ${ }^{1}$ The unscaled net charge on cation (anion) is $+1(-1)$. Figure S6a shows the that optimal intensity profiles by rescaling ionic partial charge are close to these from WAXS experiment, which is determined for the relative height of Peak 0 to be within $2 \%$ of the experimental value. The required charge rescaling factors are around 0.4 , as shown in Figure S6b. However, there is unphysical clustering between ions at these charge rescaling factors. As shown in Figure S7, for very high salt concentrations of $r=0.20$ and $r$ $=0.30$, the size of ion cluster is small (e.g., consisting of $3 \mathrm{TFSI}^{-}$for the largest cluster at $r=0.20$ ) and the distribution is symmetric between positively and negatively charged clusters. This is in sharp contrast to that reported for PEO/LiTFSI systems. ${ }^{2}$ Furthermore, rescaling down the partial charges on both $\mathrm{Li}^{+}$and $\mathrm{TFSI}^{-}$tends to reduce the interactions between ions to a larger extent than between ions and PEO. For instance, with the rescaling factors determined in Figure S6b, we observe an unphysical situation from simulation snapshot that two $\mathrm{Li}^{+}$ions are coordinated by the same PEO segments at $r=0.20$. Hence, we included the rescaling of partial charges on PEO and adjusted the force field by introducing $R_{f}$ as mentioned in the main text.
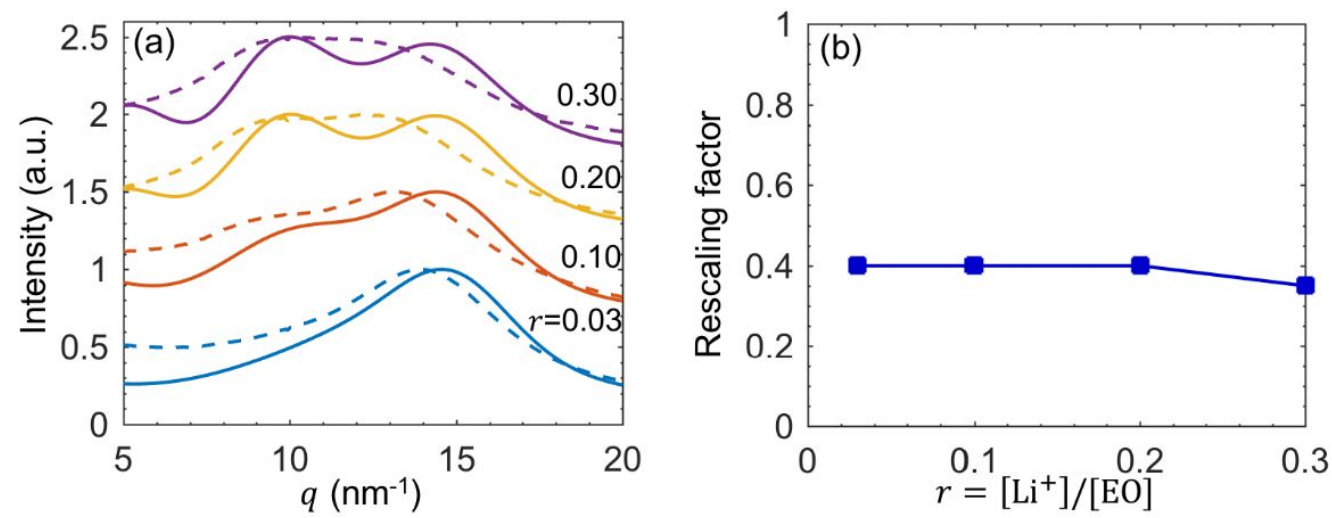

Figure S6. Rescaling of partial charges on LiTFSI. (a) The optimal intensity profiles by adjusting ionic partial charges (solid line) and the intensity profiles from WAXS experiment (dashed line) at the same salt concentration. The profiles are normalized by their maximum values and are shifted vertically by 0.5 for clarity from bottom to top. (b) The rescaling factor applied on ionic partial charges for the optimal profiles as a function of salt concentration. 
(a) $r=0.20$

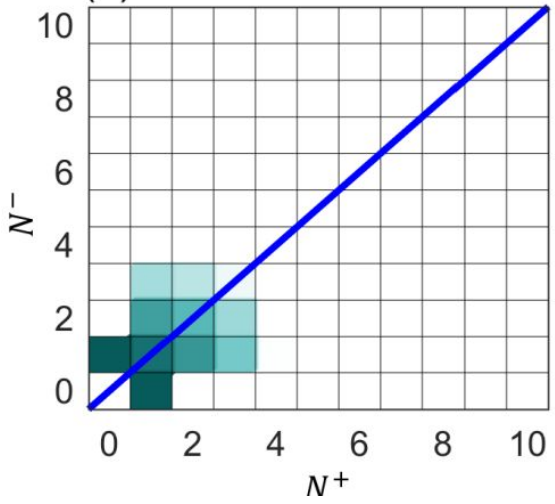

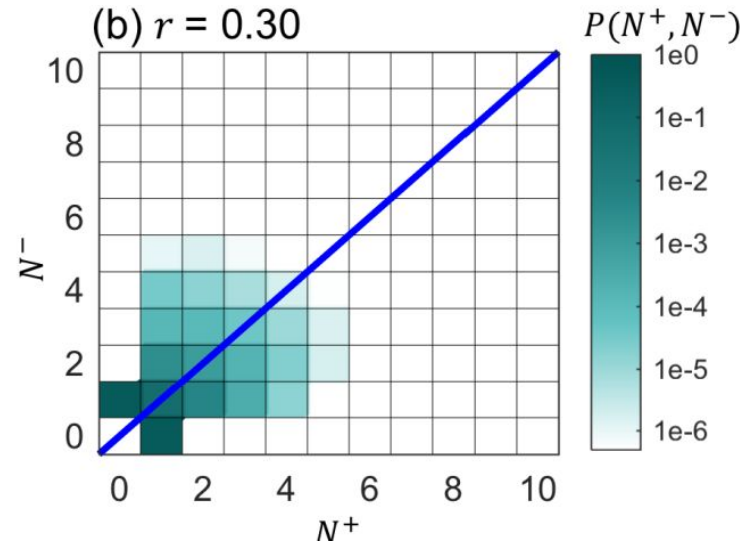

Figure S7. The probability distribution of ion cluster by rescaling partial charges on LiTFSI, $P\left(N^{+}, N^{-}\right)$ with $N^{+}$and $N^{-}$respectively the number of $\mathrm{Li}^{+}$and TFSI ${ }^{-}$within the cluster, at (a) $r=0.20$ (with rescaling factor of 0.4 ) and (b) $r=0.20$ (with rescaling factor of 0.35 ). The cluster is determined in the same way as that in the main text.

Applying $\boldsymbol{R}_{\boldsymbol{f}}$ on both LiTFSI and PEO. The optimal $R_{f}$ is determined such that the relative height of Peak 0 is within $2 \%$ of the experimental values. The profiles at these $R_{f}$ (see Figure $5 \mathrm{c}$ in the main text) is shown in Figure S8. In addition to the relative heights of the two peaks, the scattering profiles from the adjusted force field are quantitatively closer to these from WAXS experiment.

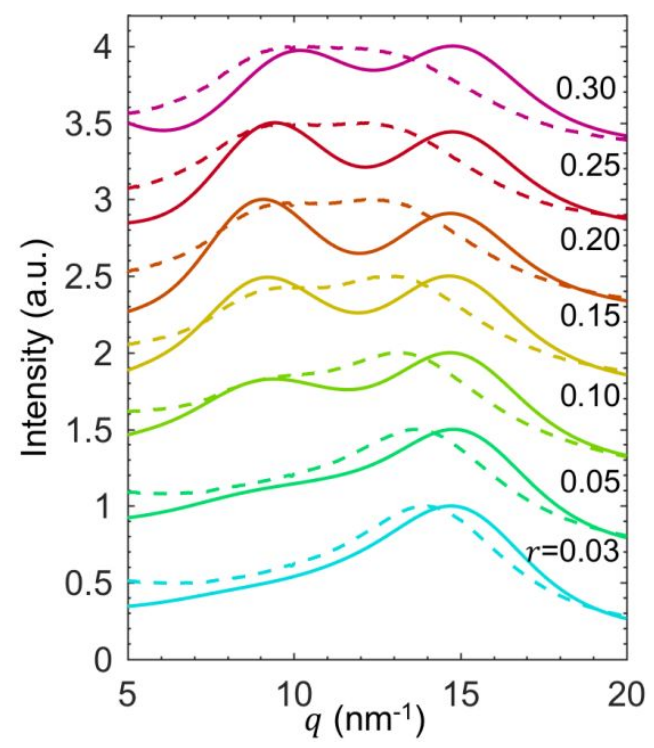

Figure S8. Intensity profiles from simulations with optimal rescaling factors $R_{f}$ (solid lines) and from experiment (dashed lines). The profiles are normalized by their maximum values and are shifted vertically by 0.5 for clarity from bottom to top. 
We rationalize the trend of $R_{f}$ presented in Figure $5 \mathrm{c}$ of the main text. $R_{f}=0.6$ at very low salt concentrations (e.g., $r=0.03$ ) indicates that the unadjusted force field overestimates the interactions between $\mathrm{Li}^{+}$and PEO segments. The need for charge rescaling at low salt concentrations can be inferred from the molecular interactions responsible for the two peaks. The overestimated interactions in the unadjusted force field result in more PEO segments being locally coordinated to one $\mathrm{Li}^{+}$ion, which leads to an increase in the intensity of the COP in $S_{P P}$ at $q \sim 6 \mathrm{~nm}^{-1}$ and a decrease in the intensity of Peak 1 at $q \sim 15.6 \mathrm{~nm}^{-1}$. The adjusted force field consequently generates a lower $\mathrm{Li}^{+}$oxygen coordination number at low salt concentration, e.g., it reduces from 6.0 to 5.1 at $r=0.03$ (see Figure $S 9$ below). This result is in better agreement with $\mathrm{Li}^{+}$coordination number obtained from all-atom simulations as well as simulations with polarizable force fields. ${ }^{1,3,4}$ The increase of $R_{f}$ above $r=0.15$ can be explained by two effects. First, the charge interactions between polymer segments and $\mathrm{Li}^{+}$ions become increasingly screened at higher salt concentrations. Second, as the TFSI- ions increasingly dominate the solvation shell of $\mathrm{Li}^{+}$with increasing salt concentration, rescaling the partial charges on the oxygen atoms of the PEO segments becomes less necessary. Together, these results elucidate the importance of improving the current force fields used in modeling ion-containing polymers by properly including the charge polarization effect.

Comparison between unadjusted and adjusted force fields. (1) Coordination number. The $\mathrm{Li}^{+}$ coordination number is determined from the radial distribution function, $g\left(r_{L i-O}\right)$, for oxygen atoms around $\mathrm{Li}^{+}$. As shown in Figure S9a, at $r=0.03\left[\mathrm{Li}^{+}\right] /[\mathrm{EO}]$ with unadjusted force field, $\mathrm{Li}^{+}$is mainly coordinated by oxygen atoms from PEO with a deep valley of $g\left(r_{L i-O}\right)$ located at $r_{L i-O}$ $=0.30 \mathrm{~nm}$. The integrated total number of oxygen atoms around $\mathrm{Li}^{+}\left(n\left(r_{\mathrm{Li}-0}\right)\right.$ in Figure S9a) increases slowly near $r_{L i-O}=0.30 \mathrm{~nm}$. This distance is used as the cutoff and a coordination number of $n(0.30 \mathrm{~nm})=6.0$ is obtained, which is the same as in previous work using the same cutoff. $^{5}$ The corresponding radial distribution functions from adjusted force field with $R_{f}=0.6$ are shown in Figure S9b. Although there is no deep valley of $g\left(r_{L i-O}\right)$ for oxygen atoms from PEO slightly beyond the first peak, $r_{L i-O}=0.30 \mathrm{~nm}$ is the location for $g\left(r_{L i-O}\right)$ of oxygen atoms from PEO to be 1 and at the valley of $g\left(r_{L i-O}\right)$ of oxygen atoms from TFSI-. Thus, the coordination number is determined using the same cutoff (obtained as 5.1). The lower than 6 coordination 
number is in better agreement with these obtained from all-atom simulations and polarizable force fields using similar cutoff. ${ }^{1,3,4}$
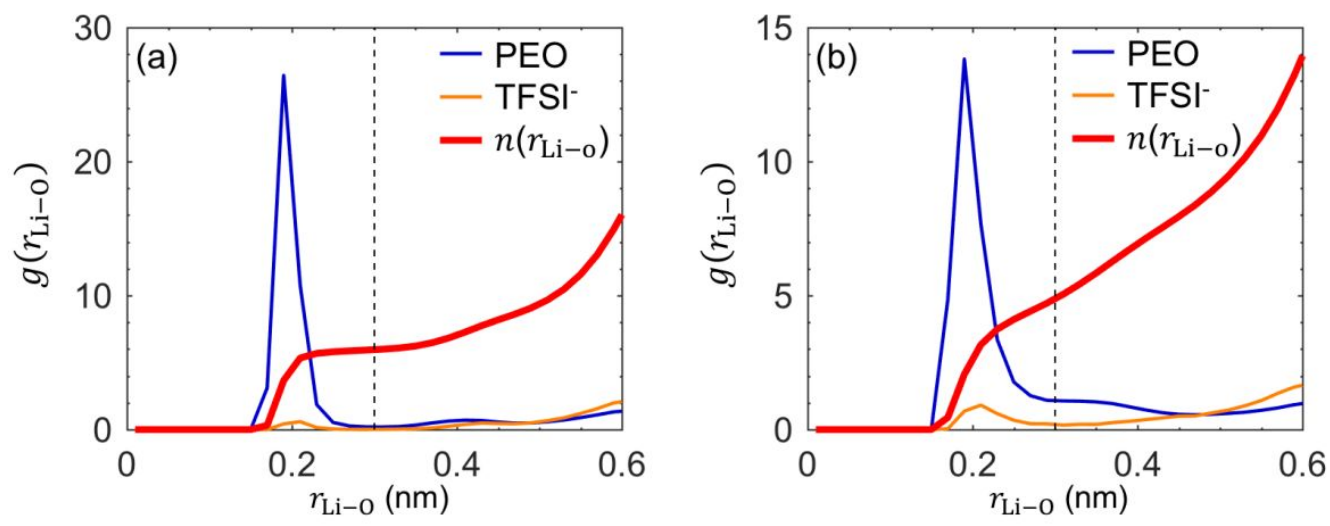

Figure S9. Radial distribution functions of oxygen atoms from PEO and TFSI along with the total number of oxygen atoms around $\mathrm{Li}^{+}\left(n\left(\mathrm{r}_{\mathrm{Li}-0}\right)\right)$ for $r=0.03\left[\mathrm{Li}^{+}\right] /[\mathrm{EO}]$ : (a) unadjusted force field; (b) adjusted force field with $R_{f}=0.6$. The dashed line denotes the position at $r_{L i-O}=0.30 \mathrm{~nm}$.

(2) Density. Figure S10 compares the density from both force fields to experimental measurement. ${ }^{6}$ The density from adjusted force field is lower than that from unadjusted force field, which is already in good agreement with experiment. On average, the density from unadjusted force is $0.6 \%$ lower than experimental values, while that from adjusted force field is $5.6 \%$ lower.

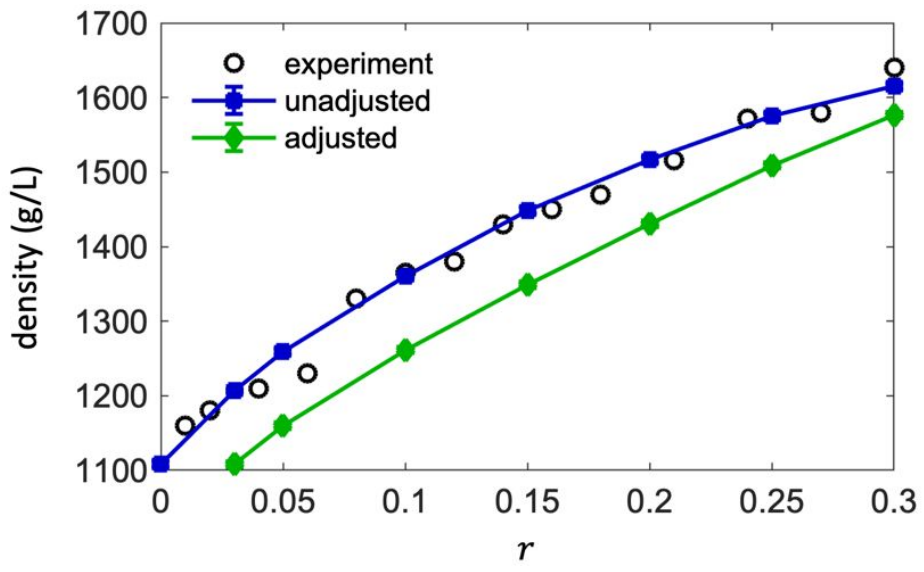

Figure S10. Density of PEO/LiTFSI at all salt concentrations from experiment (taken from ref. 6), from simulations with the unadjusted force field, and simulations with the adjusted force field.

(3) TFSI' conformation. Because the $R_{f}$ rescaling does not include the 1-4 scaling factor of TFSI', conformational distribution is affected. The torsional angle distributions are compared at $r=0.1$ 
in Figure S11, which shows that the probability distribution of the angle of S-N-S-C dihedral is affected significantly by $R_{f}$. However, in previous works of charge rescaling, the conformational distribution of anions is also affected and rarely accounted for. To rigorously maintain the conformational distribution, adjusting the scaling factor of 1-4 interaction could be further incorporated into the rescaling procedure.

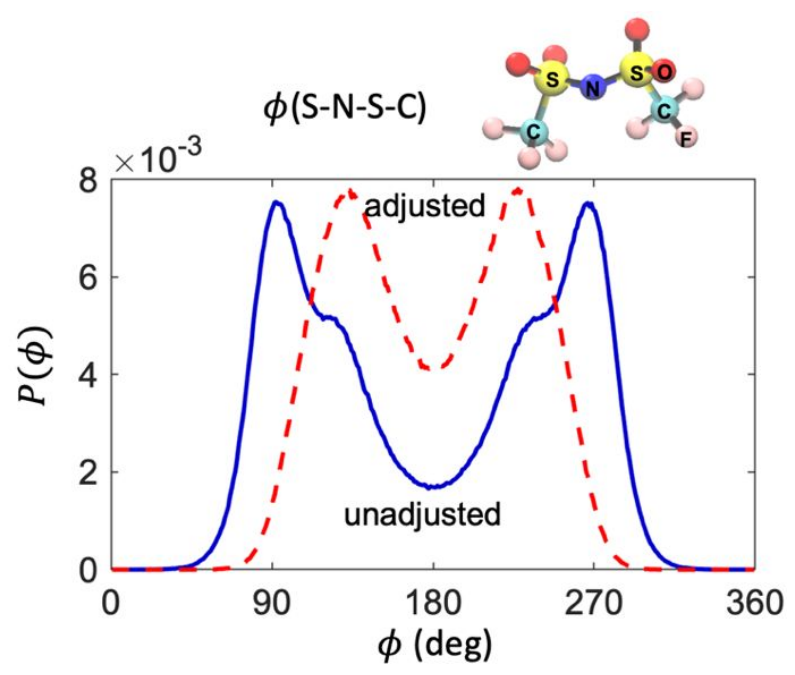

Figure S11. Torsional angle (S-N-S-C) distributions of TFSI $^{-}$with unadjusted and adjusted force field at $r=0.1$. The top sketch represents the atomic structure and chemical element of TFSI-

(4) $\mathrm{Li}^{+}$coordination from TFSI. Figure S12 compares the $g(r)$ for oxygen from $\mathrm{TFSI}^{-}$around $\mathrm{Li}^{+}$at $r=0.1$ and $r=0.2$. The first peak at $r=0.2$ is much higher than $r=0.1$, which is consistent with the larger and more negative cluster distribution in Figure $6 \mathrm{~b}$ compared with Figure $6 a$.

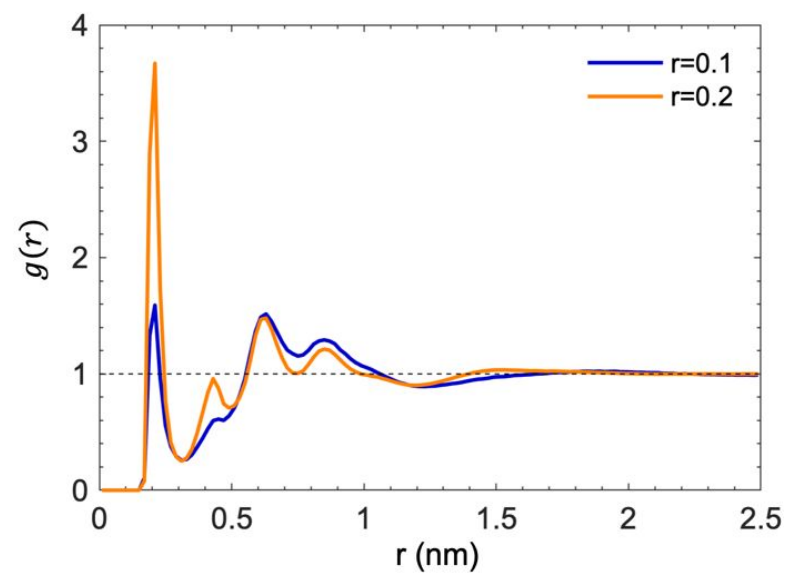

Figure S12. Radial distribution functions for oxygen from TFSI ${ }^{-}$around $\mathrm{Li}^{+}$at $r=0.1$ and $r=0.2$. 


\section{References}

1. Brooks, D. J.; Merinov, B. V.; Goddard III, W. A.; Kozinsky, B.; Mailoa, J., Atomistic description of ionic diffusion in PEO-LiTFSI: Effect of temperature, molecular weight, and ionic concentration. Macromolecules 2018, 51 (21), 8987-8995.

2. Molinari, N.; Mailoa, J. P.; Kozinsky, B., Effect of salt concentration on ion clustering and transport in polymer solid electrolytes: a molecular dynamics study of peo-litfsi. Chemistry of Materials 2018, 30 (18), 6298-6306.

3. Borodin, O.; Smith, G. D., Development of Many- Body Polarizable Force Fields for LiBattery Applications: 2. LiTFSI-Doped Oligoether, Polyether, and Carbonate-Based Electrolytes. The Journal of Physical Chemistry B 2006, 110 (12), 6293-6299.

4. Borodin, O.; Smith, G. D., Mechanism of ion transport in amorphous poly (ethylene oxide)/LiTFSI from molecular dynamics simulations. Macromolecules 2006, 39 (4), 1620-1629. 5. Webb, M. A.; Jung, Y.; Pesko, D. M.; Savoie, B. M.; Yamamoto, U.; Coates, G. W.; Balsara, N. P.; Wang, Z.-G.; Miller III, T. F., Systematic computational and experimental investigation of lithium-ion transport mechanisms in polyester-based polymer electrolytes. ACS central science 2015, l (4), 198-205.

6. Pesko, D. M.; Timachova, K.; Bhattacharya, R.; Smith, M. C.; Villaluenga, I.; Newman, J.; Balsara, N. P. Negative Transference Numbers in Poly(Ethylene Oxide)-Based Electrolytes. $J$. Electrochem. Soc. 2017, 164 (11), E3569-E3575. https://doi.org/10.1149/2.0581711jes. 